\title{
Differences in measures of the fiscal multiplier and the reduced-form vector autoregression
}

\section{Michael Donadelli, Adriana Grasso, Jean-Paul L'Huillier \& Valentina Milano}

To cite this article: Michael Donadelli, Adriana Grasso, Jean-Paul L'Huillier \& Valentina Milano (2016): Differences in measures of the fiscal multiplier and the reduced-form vector autoregression, Applied Economics Letters, DOI: 10.1080/13504851.2016.1145342

To link to this article: http://dx.doi.org/10.1080/13504851.2016.1145342

冓 Published online: 07 Mar 2016.

Submit your article to this journal $\llbracket$

Q View related articles $₫$

View Crossmark data \lceil 


\title{
Differences in measures of the fiscal multiplier and the reduced-form vector autoregression
}

\author{
Michael Donadellia, Adriana Grasso ${ }^{\mathrm{b}, \mathrm{c}}$, Jean-Paul L'Huillier ${ }^{\mathrm{c}}$ and Valentina Milano $\mathrm{o}^{\mathrm{b}, \mathrm{c}}$

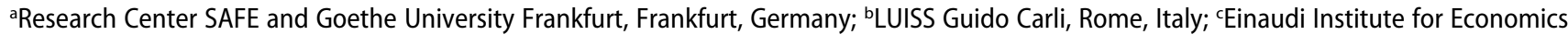 \\ and Finance (EIEF), Rome, Italy
}

ABSTRACT

The literature has recently asked whether the effects of fiscal policy vary with the state of the economy (Christiano, Eichenbaum, and Rebelo 2011; Rendahl 2014; Auerbach and Gorodnichenko 2012). We study this question in the context of vector autoregression (VAR) estimation. We show formally that, if (asymptotically) the parameters of the reduced-form VAR differ, then the dynamic effects of fiscal policy differ as well, generically and for any set of identification assumptions. Thus, in theory, the econometrician can detect these differences (either across time or space) generically just by relying on reduced-form VAR estimation.
KEYWORDS

Fiscal policy;

macroeconomic fluctuations

JEL CLASSIFICATION

E62; E32

\section{Introduction}

What determines the effects of fiscal policy in actual economies is currently a key policy issue. At some level, it seems natural to expect that there is not one constant and universal 'multiplier', but rather multipliers that depend both on the state of the economy and its underlying structure. This naturally leads to questions like: Was the multiplier different in the 1970s than in the 2000s? Is the multiplier in Germany similar to the multiplier in France?

We show that, in well-behaved cases, different reduced-form parameters values of a nonsingular VAR imply different structural dynamic responses of variables to a fiscal shock. The result is quite powerful because it applies for any set of identification restrictions. Said differently, if the parameters of the reduced-form are different (say across two different data sets), there is no identification restriction such that the obtained effects of fiscal policy are the same. The only exception is zero measure cases. That is, the statement holds generically over the parameter space.

Thus, we note that in principle the researcher interested in a yes/no answer to the questions above could skip the identification step of the structural shocks. The answer can be obtained by studying the reduced-form directly, and thus independently of identification assumptions. This is, of course, a methodological advantage. In this letter, we formally establish the asymptotical basis for this approach. This is the first step towards the construction of a test that could potentially be used in practice.

Our result is useful for two reasons. First, in practice, researchers working on fiscal policy estimation through VARs have to make an identification decision. In VARs with more than two variables, multiple possibilities for the ordering of the variables can be considered, each leading to a different Cholesky decomposition. Also, sign restrictions (Uhlig 2005) based on a priori thoughts about the sign of the impact of fiscal policy can be considered. Second, it is not ex-ante obvious that the result holds. It could well be that there exist two different sets of identification assumptions that could somehow lead to the same structural responses for some values of reduced-form parameters. Our result ensures that, generically, this is impossible.

Our article adds to a rapidly growing literature aiming to compare the fiscal multiplier over time and across countries. Auerbach, Gale, and Harris (2010) review the debate regarding the size of the fiscal multiplier and provide fresh and provocative thoughts regarding how details of the fiscal implementation and the state of the economy may influence the effectiveness of policy. Auerbach and 
Gorodnichenko (2012) provide explicit estimates of fiscal multipliers when the economy is in recession and compare them to the estimates when the economy is not. Ilzetzki, Mendoza, and Vegh (2013) show that the effects of the policy depend on key country characteristics, such as the level of development, the exchange rate regime and public indebtedness, among others.

Pioneering work by Primiceri (2005) used a VAR with time varying parameters to study the effects of monetary policy. In the context of fiscal policy, a natural step to take is to analyse following a similar approach. Some papers have already taken that route, see for instance, Kirchner, Cimadomo, and Hauptmeier (2010), Rafiq (2014), or Berg (2014). For the reasons explained above, our results below naturally fit this research agenda.

\section{Theory}

Consider the reduced-form VAR model

$$
y_{t}=B_{1} y_{t-1}+B_{2} y_{t-2}+\ldots+B_{1} y_{t-1}+u_{t}
$$

where $y_{t}$ is a $N \times 1$ vector, of which one of the variables is the measure of fiscal policy of interest. The variables in $y_{t}$ need not have any particular order. We denote by $V\left(u_{t}\right)=\sum$ the variance-covariance matrix of the innovations $u_{t}$.

Once model (1) has been estimated by ordinary least squares (OLS), we assume that identification of structural shocks can be achieved ${ }^{1}$ via a nonsingular A matrix such that

$$
u_{t}=A \varepsilon_{t}
$$

where $\varepsilon_{t}$ is the vector of structural shocks and it is such that

$$
V\left(\varepsilon_{t}\right)=I
$$

The mapping $A$ implies

$$
A A^{\prime}=\sum
$$

Definition $1 A$ set of identifying restrictions $R$ is given by $N(N-1) / 2$ equations such that $A$ is uniquely pinned down by $R$ and the equations in (2).
The following proposition establishes that if the estimated variance-covariance matrix differs across data sets, then the estimated impulse response functions (IRFs) differ as well. This proposition is useful because it suggests that the econometrician can rely on the reduced-form parameters to gauge differences in the effect of fiscal policy, no matter his stand in terms of identification.

Proposition 1 Suppose that, for two different populations, the reduced-form variance-covariance matrix $\sum$ is given by $\sum_{\mathrm{P} 1}$ and $\sum_{\mathrm{P} 2}, \sum_{\mathrm{P} 1} \neq \sum_{\mathrm{P} 2}$. Then, given two data sets, each from one of these populations, the estimated IRFs to an (exogenous) impulse to government spending differ across data sets, asymptotically and generically, under any set of identifying restrictions $R$.

Proof. For a given sample $\left\{y_{1}, \ldots, y_{T}\right\}$, write the estimated VAR

$$
y_{t}=\hat{B}_{1} y_{t-1}+\hat{B}_{2} y_{t-2}+\ldots+\hat{B}_{1} y_{t-1}+u_{t}
$$

and estimated $\sum$ by $\hat{\sum}$. Because OLS is consistent, estimates of matrix coefficients $\widehat{B}_{1}, \ldots, \widehat{B}_{1}$ of converge in probability to $B_{1}, \ldots, B_{l}$ asymptotically (for large $T$ ):

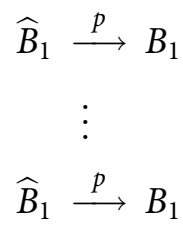

Similarly,

$$
\widehat{\sum} \stackrel{p}{\longrightarrow} \sum
$$

Thus, estimates for each of the data sets

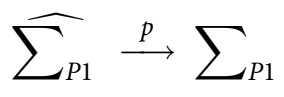

and

$$
\widehat{\sum_{P 2}} \stackrel{p}{\longrightarrow} \sum_{P 2}
$$

Without loss of generality, assume that the coefficients in both populations are the same and given by $B_{1}, \ldots, B_{l}$.

\footnotetext{
${ }^{1}$ It is well known that this assumption is restrictive because of fiscal foresight. Recently, applied researchers have argued that the inclusion of measures of fiscal expectations is a useful way to alleviate such concerns (see, for instance, the discussion in Auerbach and Gorodnichenko 2012, p. 3. See also Berg 2014.)
} 
We are interested in the impulse vector $a$ corresponding to the government shock. This vector is the column of $A$ that corresponds to the government impulse in $\varepsilon_{t}$. Following the terminology in Uhlig (2005) and using Proposition A.1 therein, this vector can be obtained as

$$
a=C \alpha
$$

where $\alpha$ is an $N$ dimensional vector of unit length and $C$ is the Cholesky decomposition of $\sum$. Because the Cholesky decomposition of $\sum$ is unique, if $\sum_{P 1} \neq$ $\Sigma_{P 2}$, the corresponding Cholesky decompositions $C_{P 1}$ and $C_{P 2}$ satisfy

$$
C_{P 1} \neq C_{P 2}
$$

From Uhlig (2005), we know that $\alpha$ is given by $R$. Our goal is to show that

$$
a_{P 1} \neq a_{P 2}
$$

Either $a_{P 1} \neq a_{P 2}$ directly from (3), or in the knifeedge case that $a_{P 1}=a_{P 2}$ we need to prove that the claim holds generically. To do so, consider an arbitrarily small perturbation $\zeta \in I R^{+}$that implies a perturbed $a_{1}^{\prime}$ given by

$$
a_{P 1}^{\prime}=\left(C_{P 1}+\zeta I\right) \alpha
$$

and $a_{P 1}^{\prime} \neq a_{P 2}$. Because the Cholesky decomposition is unique, the perturbation $\zeta$ defines uniquely a corresponding variance-covariance matrix $\sum_{P 1}^{\prime}$ with Cholesky equal to $C_{P 1}+\zeta I$, given by

$$
\sum_{P 1}^{\prime}=\left(C_{P 1}+\zeta I\right)\left(C_{P 1}+\zeta I\right) \prime
$$

The expression shows that $\sum_{P 1}^{\prime}$ can be made arbitrarily close to $\sum_{P 1}$ by taking $\zeta$ small. To conclude, generically the IRFs to a government spending shock differ, as claimed. This completes the proof.

For reasons of space, we have focused on the case where parameters of the variance-covariance matrix differ. A similar argument can be given for differences on parameters in $\widehat{B}_{1}, \ldots, \widehat{B}_{1}$. To see this, notice that for large $T$, the IRFs are

$$
y_{t}=A \varepsilon_{t}, y_{t+1}=B_{1} A \varepsilon_{t,} y_{t+2}=B^{2}{ }_{1} A \varepsilon_{t}+B_{2} A \varepsilon_{t}, \ldots
$$

Thus, a difference in $\widehat{B}_{1}, \ldots, \widehat{B}_{1}$. between populations will also imply, generically, a difference in the IRFs.
A remark is important regarding the precise meaning of Proposition 1 holding generically. As it is clear in the proof, this means that the result applies to open and dense sets of parameters. As such, it is possible to find examples in which the result does not hold. But all those examples have to be knife-edge cases, and thus in some sense misleading. This is the claim in the Proposition.

\section{Conclusion}

We have proven a theorem relating the parameters of a reduced-form VAR and the IRFs to an identified fiscal policy shock. The theorem shows that, independently of identification assumptions, different reduced-form parameters induce (asymptotically and generically) different IRFs to a fiscal policy shock. Based on this result, a researcher solely interested in differences between fiscal policy multipliers across time, states or countries can skip the identification step as a first pass to the data. A formal derivation of a statistical test based on this idea is left for future work.

\section{Acknowledgements}

We thank Pierpaolo Benigno, Daniele Terlizzese and LUISS Guido Carli seminar participants for useful comments. Grasso and Milano thank the Research Center SAFE for hospitality and support during the preparation of this article.

\section{Disclosure statement}

No potential conflict of interest was reported by the authors.

\section{References}

Auerbach, A., W. G. Gale, and B. H. Harris. 2010. “Activist Fiscal Policy." Journal of Economic Perspectives 24 (4): 141-164. doi:10.1257/jep.24.4.141.

Auerbach, A., and Y. Gorodnichenko. 2012. "Measuring the Output Responses to Fiscal Policy." American Economic Journal-Economic Policy 4 (2): 1-27. doi:10.1257/pol.4.2.1.

Berg, T. O. 2014. "Time Varying Fiscal Multipliers in Germany.” MPRA Working Paper 57223.

Christiano, L., M. Eichenbaum, and S. Rebelo. 2011. "When Is the Government Spending Multiplier Large?" Journal of Political Economy 119 (1): 78-121. doi:10.1086/659312. 
Ilzetzki, E., E. G. Mendoza, and C. A. Vegh. 2013. "How Big (Small) are Fiscal Multipliers?" Journal of Monetary Economics 60: 239-254. doi:10.1016/j.jmoneco.2012.10.011.

Kirchner, M., J. Cimadomo, and S. Hauptmeier. 2010. "Transmission of Government Shocks in the Euro Area: Time Variation and Driving Forces.” ECB Working Paper 1219.

Primiceri, G. 2005. "Time Varying Structural Vector Autoregressions and Monetary Policy." Review of Economic Studies 72 (3): 821-852. doi:10.1111/roes.2005.72.issue-3.
Rafiq, S. 2014. "UK Fiscal Multipliers in the Postwar Era: Do State Dependent Shocks Matter?” CESifo Economic Studies 60 (1): 213-245. doi:10.1093/cesifo/ift011.

Rendahl, P. 2016. Fiscal Policy in an Unemployment Crisis Review of Economic Studies. Forthcoming.

Uhlig, H. 2005. "What are the Effects of Monetary Policy on Output? Results from an Agnostic Identification Procedure." Journal of Monetary Economics 52: 381-419. doi:10.1016/j. 